\title{
Apendicite Simulando Malformação Mülleriana em Crianças: Relato de Caso
}

\author{
Chronic Appendicitis Simulating Müllerian Malformation in Children: Case Report \\ Marco Fábio Prata Lima, Eddie Fernando Cândido Murta, Adriana Cartafina Perez-Bóscollo \\ Paulo José Maluf, Ângelo Gustavo Zucca Matthes, Aldo Benjamin Rodrigues Barbosa
}

\section{RESUM0}

A apendicite crônica pode simular diversas situações clínicas, dificultando a conduta clínica. Descrevemos neste trabalho um caso de uma menina de 9 anos com dor abdominal há 13 meses, sendo visualizada massa expansiva no baixo ventre após exames de ultra-som e tomografia computadorizada abdominal e laparoscopia diagnóstica. Houve enorme dificuldade diagnóstica, pois o quadro clínico, laboratorial e imaginológico não foi conclusivo, levando a várias hipóteses diagnósticas. O diagnóstico definitivo de plastrão apendicular ocorreu somente após laparotomia exploradora. Discutem-se neste estudo vários diagnósticos diferenciais quando da presença de massa formada por plastrão apendicular com enfoque nas malformações ginecológicas, endometriose, neoplasias e pseudotumores.

PALAVRAS-CHAVE: Apendicite crônica. Plastrão apendicular. Massa abdominal.

Introdução

O plastrão apendicular é um dos diagnósticos que podem provocar no médico o sentimento de incerteza acerca de uma conduta precisa a ser tomada ${ }^{1,2}$. Marque et al. $^{3}$ usaram a indagação no título de seu artigo para enfatizar a surpresa em relação ao diagnóstico de um abscesso apendicular - "What diagnosis? Appendicular abscess". Moser et al. ${ }^{4}$ escreveram sobre os surpreendentes diagnósticos referentes às massas inflamatórias aparentes da fossa ilíaca direita, de causas extragenitais e genitais, ambos ilustrando bem a possibilidade de dificuldade ou, até mesmo, erro de raciocínio em certos quadros clínicos. Estes quadros são ainda mais inconsistentes na mulher ${ }^{1}$.

Inúmeras patologias ginecológicas que simulam quadro de abdome agudo ginecológico devem ser pesquisadas na presença de massa em topografia anexial direita. Cistos anexiais

Trabalho realizado na Disciplina de Ginecologia e Obstetrícia da Faculdade de Medicina do Triângulo Mineiro (FMTM).

Correspondência: Marco Fábio Prata Lima - Disciplina de Ginecologia e Obstetrícia da Faculdade de Medicina do Triângulo Mineiro

Av. Getúlio Guaritá, S/N

38.025-440 - Uberaba-MG

e-mail: m_fabio@mednet.com.br hemorrágicos ou torcidos, gravidez ectópica, abscesso tubo-ovariano, miomas uterinos e neoplasia ovariana primária ou metastática são alguns exemplos. Entre as causas extragenitais destacam-se os tumores mesenquimais do retroperitôneo pélvico e outras várias patologias que mimetizam quadros clínicos que comumente se aglutinam e perpetuam a propedêutica ${ }^{2}$.

$\mathrm{Na}$ criança e na adolescente com massa anexial, obrigatória se faz a distinção com as malformações müllerianas, principalmente aquelas que cursam com obstrução ou semi-obstrução ao fluxo menstrual, como os septos transversos de vagina, o corno uterino rudimentar e diversos outros defeitos müllerianos ${ }^{5}$. Entre as patologias inflamatórias do trato gastrointestinal destacamse a doença diverticular dos cólons, o abscesso apendicular, a doença de Crohn e a tuberculo$\mathrm{se}^{4}$.

Entre estas últimas o plastrão apendicular ou apendicite hiperplásica é o que mais permite uma série de raciocínios diferenciais ou paralelos que comumente ofuscam a elucidação clínica. Na infância, seu diagnóstico é mais complexo devido à ausência de exames laboratoriais ou radiológicos específicos que o diferencie das doenças digestivas ou ginecológicas, especialmente das malformações dos ductos de Müller ${ }^{4}$. 
Descrevemos aqui um caso de plastrão apendicular de difícil diagnóstico, atestando as dificuldades clínicas descritas na literatura e que exigiu extensa semiologia.

\section{Relato de Caso}

MRS, branca, 9 anos, natural e procedente de Uberaba-MG.

A paciente procurou o Ambulatório de $\mathrm{Pe}$ diatria da FMTM queixando-se de dor abdominal há 20 dias, contínua, no hipogástrio, de moderada intensidade, associada a tontura, náuseas, vômitos, alguns episódios de febre baixa e emagrecimento de $3 \mathrm{~kg}$ e tendo já feito uso de antieméticos e antibióticos por via oral, apresentando, a seguir, discreta melhora do quadro. Ao exame físico mostrava discreta dor à palpação do hipogástrio. Seus exames de urina I, urocultura e parasitológico de fezes eram normais. A avaliação ultra-sonográfica do abdomem revelou anormalidades (Figura 1). Foi medicada apenas com sintomáticos. Quinze dias mais tarde, procurou o Ambulatório de Cirurgia Pediátrica, com as mesmas queixas anteriormente referidas. Nova ultra-sonografia foi realizada, sendo visualizada grande imagem cística de paredes finas e conteúdo anecóico, ocupando todo o fundo de saco posterior, deslocando o útero anteriormente, medindo $7,4 \times 4,6 \times 5,8 \mathrm{~cm}$ de diâmetro (volume $=91 \mathrm{~mL}$ ). Foram solicitados, então, os seguintes marcadores tumorais: CA-125, CA-15.3, CA-19.9, a-fetoproteína, CEA, FSH, LH, progesterona e $\beta$-HCG. Todos estavam dentro dos valores normais. Optou-se inicialmente por uma conduta expectante e observação clínica. Dois meses se passaram e a paciente procurou novamente o pronto-socorro pediátrico com quadro clínico semelhante, associado agora a leucorréia, disúria ao final das micções e dores lombares. Ao exame físico, apresentava-se em bom estado geral, porém com abaulamento infra-umbilical, dor à palpação da fossa ilíaca direita, hipogástrio e fossa ilíaca esquerda e contratura voluntária dessa região. Os sinais de Blumberg e Lennander eram negativos. A ultra-sonografia evidenciou massa expansiva complexa na região mediana da pelve, irregular e lobulada, medindo 5,3 x 4,2 $\mathrm{x} 7,5 \mathrm{~cm}$ (volume $=87,5 \mathrm{~mL}$ ), sugestiva de hematométrio (Figura 2). Nova ultrassonografia, dois dias após, foi então realizada e evidenciouse regressão do volume da massa antes visualizada em topografia uterina (Figura 3). A tomografia computadorizada mostrou útero com dimensões aumentadas e hipodensidade em seu interior, sem sinais de invasão adjacente, e ectasia ureterocalicial bilateral, compatíveis com hematométrio e compressão de ureteres (Figura 4). As radiografias de tórax e abdome foram normais. Sedada, a paciente foi levada à colpovirgoscopia, que evidenciou vagina e colo uterino normais, sendo no mesmo ato realizado videolaparoscopia, que constatou pelve congelada, com aderências que impossibilitaram a visualização dos órgãos pélvicos. Levada, então, no mesmo ato à laparotomia exploradora, foi visualizado útero de volume normal e anexos aderidos às alças intestinais no fundo de saco posterior, aparentemente normais. O apêndice retrocecal formava plastrão em fundo de saco posterior, com características inflamatórias crônicas. Foi realizada apendicectomia.

O estudo anatomopatológico revelou apendicite com flegmão. A paciente recebeu alta hospitalar 6 dias após em bom estado geral e evolui sem queixas.

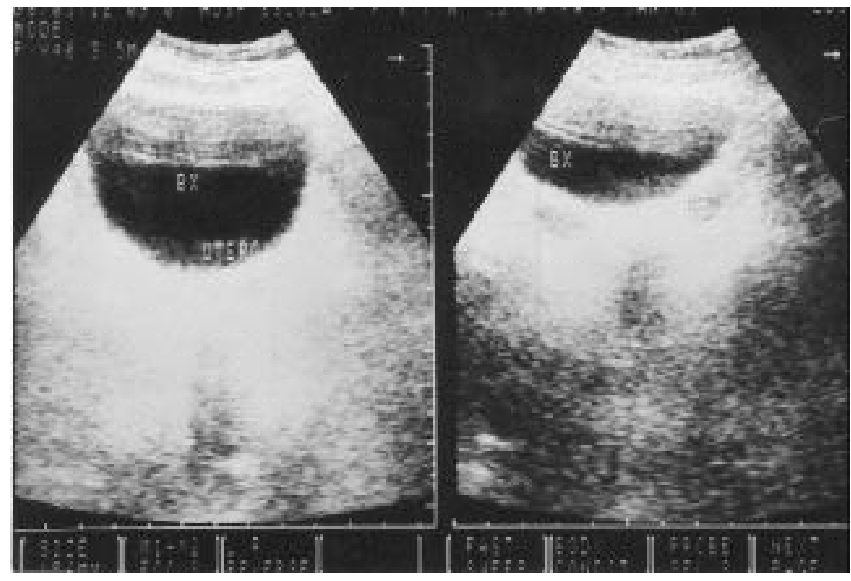

Figura 1-Ultra-sonografia pélvica mostrando imagens uterina e anexial compatíveis com a idade dapaciente.

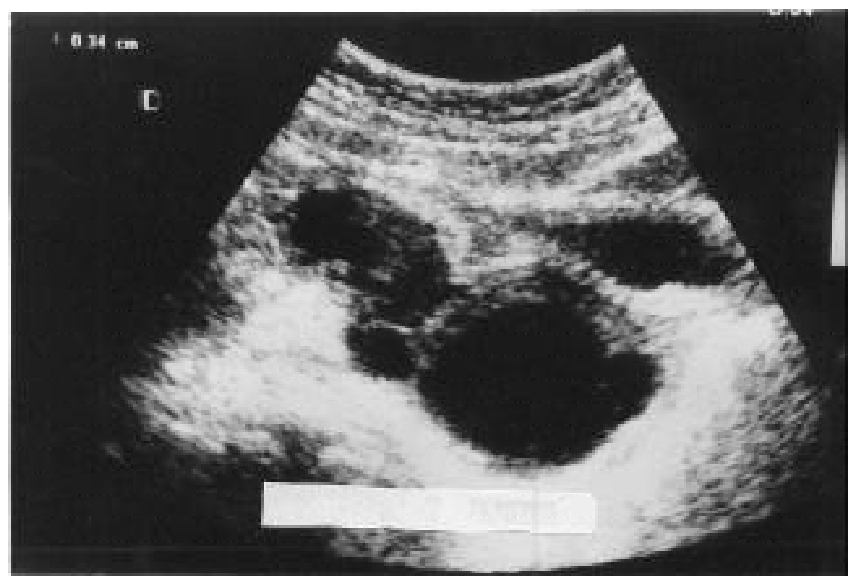

Figura 2-Ultra-sonografia pélvica evidenciando volumosa massa em topografia uterina sugestiva de hematométrio. 


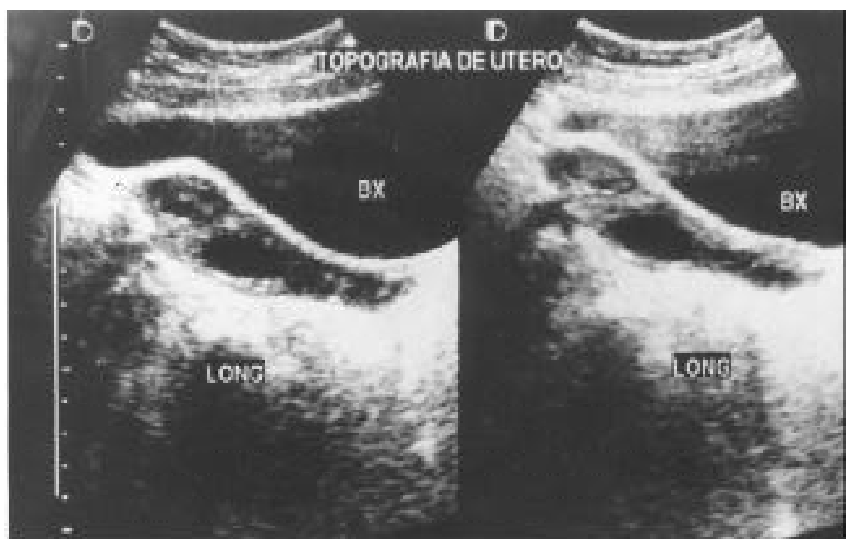

Figura 3 - Ultra-sonografia pélvica mostrando regressão espontânea da massa vista em exame anterior.

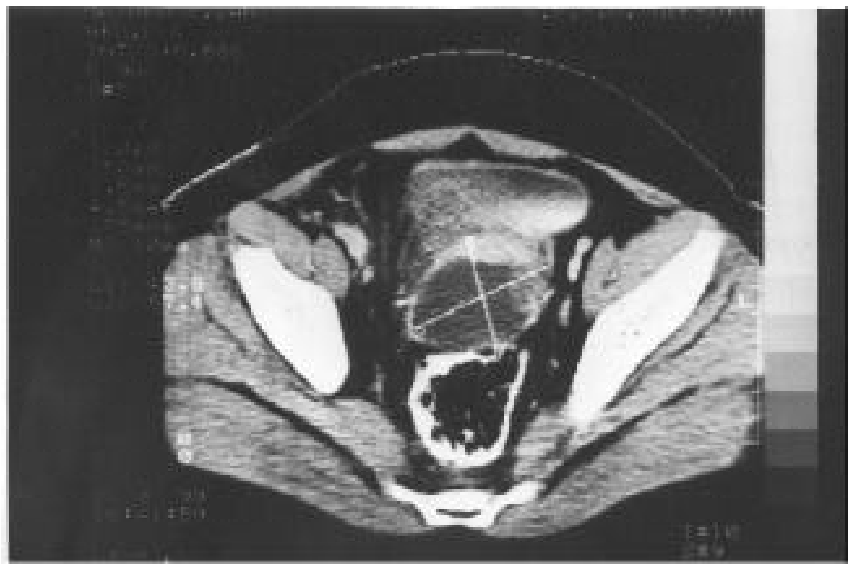

Figura 4 - Tomografia computadorizada da pelve revelando massa pélvica e provável ectasia ureteral.

\section{Discussão}

A apendicite crônica oferece por vezes enormes dificuldades diagnósticas pois perdem-se os parâmetros patognomônicos de infecção aguda mais comumente vistos ${ }^{6,7}$. Assim como evidenciado nesse relato, a paciente procurou auxílio médico, apresentando um quadro clínico atípico, com dor abdominal esporádica no hipogástrio. Ela não apresentou, em nenhum momento, irritação peritoneal, sendo ausentes em todos os seus atendimentos em ambulatórios ou prontosocorro, os característicos sinais de Blumberg e Lennander. Apresentou apenas episódios fugazes de febre e vômito. O diagnóstico tornou-se ainda mais difícil devido ao sexo da paciente e a idade em que se encontrava, o que instigou raciocínios de ordem ginecológica, como hímen imperfurado, útero bicorno, vagina em fundo cego ou outras malformações dos ductos de Müller ou alterações morfofuncionais ginecológicas como cisto ou torção anexial, neoplasias ginecológicas ou ainda doença inflamatória pélvica e endometriose, todas supostamente reforçadas pelos hemogramas e estudos de imagem pouco definidos.

As anomalias de fusão dos ductos de Müller podem determinar quadros variáveis, desde útero duplo, com ou sem septação vaginal, até discretos septos intra-uterinos. As variantes mais freqüentes englobam o útero unicorno com ou sem corno rudimentar que, clinicamente, quando não comunicante pode causar dor, dismenorréia, massa pélvica, refluxo de sangue abdominal e endometriose. Já o útero bicorno que exibe graus variáveis de fusão e que associa septos longitudinais completos ou não dificilmente determina ocorrência de massa pélvica ${ }^{5}$.

A propedêutica imaginológica como a tomografia computadorizada subseqüente ao ultra-som poderia facilitar o diagnóstico de uma apendicite epiplóica ${ }^{8}$, contudo tal não aconteceu nesse caso. No entanto, estes exames foram úteis para mostrar o acometimento das vias renais devido à compressão dos ureteres, que ocasionou ectasia ureterocalicial bilateralmente o que já fora descrito primeiramente ${ }^{9}$.

Conclui-se que, diante de uma criança do sexo feminino com dor na fossa ilíaca direita com ou sem formação de plastrão, é imperativa a investigação extensa, uma vez que os diagnósticos diferenciais são de várias naturezas e comumente exigem semiologia complexa e raciocínio clínico apurado.

\section{SUMMARY}

Chronic appendicitis might simulate several diseases, making clinical management difficult. We report the case of a 9-year-old girl with an abdominal pain for 13 months. Ultrasound and tomography showed an expansive mass in the lower abdomen. It was extremely difficult to establish the diagnosis because the symptoms, laboratory results and images were inconclusive. The definitive diagnosis occurred only after laparotomy. We discuss in the present article several differential diagnoses for chronic appendicitis, mainly gynecological malformations, endometriosis, neoplasias and pseudotumors.

KEY WORDS: Chronic appendicitis. Abdominal mass. Differential diagnosis. 


\section{Referências}

1. Alaminos Mingorance M, Castejon Casado J, Valladares Mendias JC, Villegas Rubi M, Sanchez Lopez-Tello C. Deferred appendectomy in a case of appendiceal mass. Cir Pediatr 1998; 11:16870 .

2. De Mestier du Bourg P. Diagnosis of a pelvic mass. J Chir (Paris) 1998; 135:168.

3. Marque B, Montaigut JY, Wafflart E, Pons F, Jeanbourquin D. What diagnosis? Appendicular abscess. J Radiol 1998; 79:65-7.

4. Moser JJ, Barras JP, Bauer HU. Diagnostic surprises in apparently inflammatory masses of the right iliac fossa. Helv Chir Acta 1994; 60:653-6.

5. Mieli MPA, Yokoshi K, Vilaça Júnior PR, Matsunaga P. Hímen imperfurado: diagnóstico diferencial, complicações e tratamento. Femina 1999; 27:175-6.

6. Platel JP, Terrier JP, Farthouard P, Thouard H, Flandrin P. Appendicular pseudo-tumors: unusual diagnosis. Ann Chir 1998; 52:326-30.

7. Montero Sánchez M, Gomez Tellado M, Mendes Gallart R, et al. Ureterohidronefrosis secundaria a abscesso apendicular. Arch Esp Urol 1997; 50:972-5.

8. Pellerito JS, McCarthy SM, Doyle MB, Glickman MG, DeCherney AH. Diagnosis of uterine anomalies: relative accuracy of MR imaging, endovaginal sonography and hysterosalpingography. Radiology 1992; 183:795-800.

9. Nycum LR, Moss H, Adams JQ, Macri CI. Asymptomatic intussusception of the appendix due to endometriosis. South Med J 1999; 92:5245.

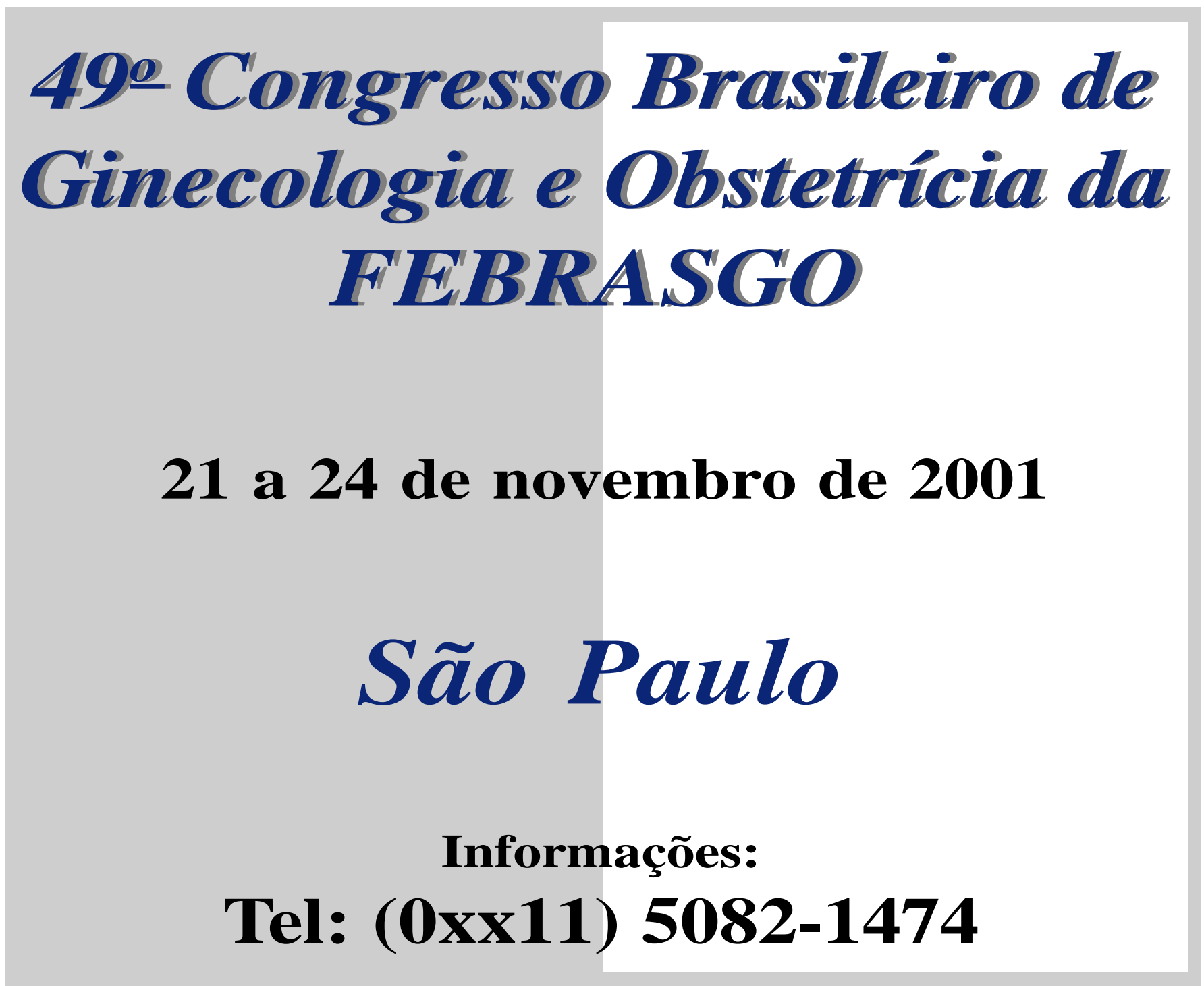

